\title{
A recursive update cubature Kalman filter
}

\author{
Yonggang Zhang, Gang Wang ${ }^{\mathrm{a}}$ and Ning Li \\ School of Automation, Harbin Engineering University, Harbin 150001, China; \\ awanggang2016@126.com
}

Keywords: Recursive update, cubature Kalman filter, iterate cubature Kalman filter.

\begin{abstract}
This paper investigates the use of recursive update and proposes a recursive update cubature Kalman filter (RUCKF). Merit of recursive update is a better assimilation of measurement information and realization of nonlinear update on the state. The proposed algorithm is shown to achieve superior performance in terms of efficiency and accuracy when compared to cubature Kalamn filter (CKF), iterated cubature Kalman filter (ICKF) and recursive update filter based on extended Kalman filter (RUF). Illustrative simulation has been provided to prove this conclusion.
\end{abstract}

\section{Introduction}

For nonlinear system, it is difficult to give out a closed-form recursive state estimation. To solve this problem, the assumption of a jointly Gaussian distributed state and measurement is used. Taking the classical Kalman filter (KF) as a benchmark, all KF approximations [1] including the extended Kalman filter (EKF) [2], unscented Kalman filter (UKF) [3], cubature Kalman filter (CKF) [4], Gaussian-Hermite filter (GHF) [5] and so on are implemented based on this assumption. Unfortunately, it is shown that the KF approximations would fail to estimate the state accurately when they are encountered with particular type of nonlinearities [6, 7]. In [6], it points out that the joint Gaussian assumption would result in a poor approximation of the true mean and variance, which may cause a significant degradation in estimation performance or even a divergence of the estimator. Analogous conclusions have been drawn in [8]. It claims that the optimality of linear minimum mean square error (LMMSE) estimator in linear system doesn't guarantee good performance for certain measurement nonlinearity. Then a recursive update filter (RUF) was proposed and its key idea is that the measurement information is incorporated recursively while following the curvature of the nonlinear measurement.

Subjected to the drawbacks of EKF, such as the linearization assumption, failure in strong nonlinearities, inconsistent estimate of the error covariance and calculation of Jacobian matrices, the filter performance of RUF proposed in [8] is limited. Inspired by [8], the recursive update idea is generalized into cubature transform (CT) and a recursive update cubature Kalman filter (RUCKF) is proposed in this paper. Compared with the resemble algorithms, CKF, iterated cubature Kalman filter (ICKF) and RUF, the proposed method outperforms them markedly.

The remaining of this paper is organized as follows. The GF framework is reviewed and the investigated problem is formulated in Section 2. In Section 3, we discuss the difference between iterate update and recursive update, then the recursive update (RU) idea is generalized into CT and the RUCKF is proposed. Simulation is given in Section 4 and conclusions are drawn in Section 5.

\section{Gaussian Filter}

Consider the following discrete time nonlinear state-space model

$$
\begin{aligned}
& \boldsymbol{x}_{k+1}=f\left(\boldsymbol{x}_{k}\right)+\boldsymbol{w}_{k} . \\
& \mathbf{z}_{k+1}=h\left(\boldsymbol{x}_{k+1}\right)+\boldsymbol{v}_{k+1} .
\end{aligned}
$$

where $\boldsymbol{x}_{k} \in \boldsymbol{R}^{n}, \mathbf{z}_{k+1} \in \boldsymbol{R}^{m}$ are the state and measurement vector, $n, m$ are dimensions of state-space and measurement, respectively. $f(\cdot)$ is the state transition function and $h(\cdot)$ is the measurement 
function. The process noise $\boldsymbol{w}_{k}$ and measurement noise $\boldsymbol{v}_{k}$ are zero-mean Gaussian noises which satisfies $E\left[\boldsymbol{w}_{k} \boldsymbol{w}_{j}^{T}\right]=\boldsymbol{Q}_{k} \delta_{k, j}$ and $E\left[\boldsymbol{v}_{k+1} \boldsymbol{v}_{j+1}^{T}\right]=\boldsymbol{R}_{k+1} \delta_{k+1, j+1}$, where $\delta_{k, j}$ is the Kronecker-delta function.

According to the LMMSE rule, filters are designed to estimate conditional mean $E\left[x_{k+1} \mid Z_{k}\right]$, $E\left[\boldsymbol{z}_{k+1} \mid \boldsymbol{Z}_{k}\right]$ and $E\left[\boldsymbol{x}_{k+1} \mid \boldsymbol{Z}_{k+1}\right]$ with corresponding covariance $\boldsymbol{P}_{k+1 \mid k}, \boldsymbol{P}_{z, k+1 k}, \boldsymbol{P}_{x z, k+1 k}$ and $\boldsymbol{P}_{k+1 k+1}$ based on the known measurement sequence $\boldsymbol{Z}_{k+1}=\left\{\mathbf{z}_{1}, \mathbf{z}_{2}, \cdots, \mathbf{z}_{k+1}\right\}$ at time $k+1$.

The framework of Gaussian filter is given by [9]

$$
\left\{\begin{array}{l}
\hat{\boldsymbol{x}}_{k+1 \mid k+1}=\hat{\boldsymbol{x}}_{k+1 \mid k}+\boldsymbol{K}_{k+1}\left(\boldsymbol{z}_{k+1}-\hat{\boldsymbol{z}}_{k+1 \mid k}\right) \\
\boldsymbol{P}_{k+1 \mid k+1}=\boldsymbol{P}_{k+1 \mid k}-\boldsymbol{K}_{k+1}\left(\boldsymbol{P}_{z, k+1 \mid k}\right)^{-1} \boldsymbol{K}_{k+1}^{T} \\
\boldsymbol{K}_{k+1}=\boldsymbol{P}_{x z, k+1 \mid k}\left(\boldsymbol{P}_{z, k+1 \mid k}\right)^{-1}
\end{array} .\right.
$$

where

$$
\left\{\begin{array}{l}
\hat{\boldsymbol{x}}_{k+1 \mid k}=E\left[f\left(\boldsymbol{x}_{k}\right) \mid \mathbf{Z}_{k}\right] \\
\boldsymbol{P}_{k+1 \mid k}=E\left[\left(\boldsymbol{x}_{k+1}-\hat{\boldsymbol{x}}_{k+1 \mid k}\right)\left(\boldsymbol{x}_{k+1}-\hat{\boldsymbol{x}}_{k+1 \mid k}\right)^{T} \mid \mathbf{Z}_{k}\right] \\
\hat{\boldsymbol{z}}_{k+1 \mid k}=E\left[h\left(\boldsymbol{x}_{k+1}\right) \mid \mathbf{Z}_{k}\right] \\
\boldsymbol{P}_{z, k+1 \mid k}=E\left[\left(\boldsymbol{z}_{k+1}-\hat{\mathbf{z}}_{k+1 \mid k}\right)\left(\boldsymbol{z}_{k+1}-\hat{\mathbf{z}}_{k+1 \mid k}\right)^{T} \mid \mathbf{Z}_{k}\right] \\
\boldsymbol{P}_{x z, k+1 \mid k}=E\left[\left(\boldsymbol{x}_{k+1}-\hat{\boldsymbol{x}}_{k+1 \mid k}\right)\left(\mathbf{z}_{k+1}-\hat{\mathbf{z}}_{k+1 \mid k}\right)^{T} \mid \mathbf{Z}_{k}\right]
\end{array} .\right.
$$

From (3)-(4), we know that the state prediction $\hat{\boldsymbol{x}}_{k+1 \mid k}$ is corrected by measurement $\mathbf{z}_{k+1}$ once based on the rule of LMMSE. Unfortunately, it is illustrated in $[6,8]$ that the LMMSE estimators couldn't get a good performance for certain measurement nonlinearities. In such a situation, more attentions should be focused on how to exploit the observation information in nonlinear measurement.

\section{Recursive update cubature Kalman filter (RUCKF)}

Recursive Update (RU). In the procedure of RU, the optimal gain varies following the curvature of the nonlinear measurement function. At every update step, measurement is used to correct the state prediction to a scale instead of all. Referred to [8], the procedure of RU is given as follows.

$$
\begin{aligned}
\boldsymbol{C}_{k+1}^{(0)}=0, \boldsymbol{P}_{k+1 \mid k+1}^{(0)}= & \boldsymbol{P}_{k+1 \mid k}, \hat{\boldsymbol{x}}_{k+1 \mid k+1}^{(0)}=\hat{\boldsymbol{x}}_{k+1 \mid k} \\
\text { for } i=1: N & \\
\boldsymbol{H}_{k+1}^{(i-1)}= & \partial h /\left.\partial \boldsymbol{x}\right|_{\boldsymbol{x}=\boldsymbol{x}_{k+1 k+1}^{(i-1)}} \\
\boldsymbol{K}_{k+1}^{(i)}= & \frac{1}{N-i+1}\left(\boldsymbol{P}_{k+1 k+1}^{(i-1)} \boldsymbol{H}_{k+1}^{(i-1) T}+\boldsymbol{C}_{k+1}^{(i-1)}\right)\left(\boldsymbol{H}_{k+1}^{(i-1)} \boldsymbol{P}_{k+1 k+1}^{(i-1)} \boldsymbol{H}_{k+1}^{(i-1) T}+\boldsymbol{R}_{k+1}+\boldsymbol{H}_{k+1}^{(i-1)} \boldsymbol{C}_{k+1}^{(i-1)}+\boldsymbol{C}_{k+1}^{(i-1) T} \boldsymbol{H}_{k+1}^{(i-1) T}\right)^{-1} \\
\hat{\boldsymbol{x}}_{k+11 k+1}^{(i)}= & \hat{\boldsymbol{x}}_{k+1 \mid k+1}^{(i-1)}+\boldsymbol{K}_{k+1}^{(i)}\left(\mathbf{z}_{k+1}-h\left(\hat{\boldsymbol{x}}_{k+1 \mid k+1}^{(i-1)}\right)\right) \\
\boldsymbol{P}_{k+1 \mid k+1}^{(i)}= & \left(\boldsymbol{I}_{n \times n}-\boldsymbol{K}_{k+1}^{(i)} \boldsymbol{H}_{k+1}^{(i-1)}\right) \boldsymbol{P}_{k+1 \mid k+1}^{(i-1)}\left(\boldsymbol{I}_{n \times n}-\boldsymbol{K}_{k+1}^{(i)} \boldsymbol{H}_{k+1}^{(i-1)}\right)^{T}+\boldsymbol{K}_{k+1}^{(i)} \boldsymbol{R}_{k+1} \boldsymbol{K}_{k+1}^{(i) T}- \\
& \left(\boldsymbol{I}_{n \times n}-\boldsymbol{K}_{k+1}^{(i)} \boldsymbol{H}_{k+1}^{(i-1)}\right) \boldsymbol{C}_{k+1}^{(i-1)} \boldsymbol{K}_{k+1}^{(i) T}-\boldsymbol{K}_{k+1}^{(i)} \boldsymbol{C}_{k+1}^{(i-1) T}\left(\boldsymbol{I}_{n \times n}-\boldsymbol{K}_{k+1}^{(i)} \boldsymbol{H}_{k+1}^{(i-1)}\right)^{T} \\
\boldsymbol{C}_{k+1}^{(i)}= & \left(\boldsymbol{I}-\boldsymbol{K}_{k+1}^{(i)} \boldsymbol{H}_{k+1}^{(i-1)}\right) \boldsymbol{C}_{k+1}^{(i-1)}-\boldsymbol{K}_{k+1}^{(i)} \boldsymbol{R}_{k+1}
\end{aligned}
$$

end for

$$
\hat{\boldsymbol{x}}_{k+1 \mid k+1}=\hat{\boldsymbol{x}}_{k+1 \mid k+1}^{(N)}, \boldsymbol{P}_{k+1 \mid k+1}=\boldsymbol{P}_{k+1 k+1}^{(N)}
$$

where $N$ is the number of recursions which is a user-defined parameter, $\boldsymbol{C}_{k+1}$ represents the cross-covariance between the state $\boldsymbol{x}_{k+1}$ and measurement noise $\boldsymbol{v}_{k+1}$ defined as $\boldsymbol{C}_{k+1}=E\left[\left(\boldsymbol{x}_{k+1}-\hat{\boldsymbol{x}}_{k+1 \mid k+1}\right) \boldsymbol{v}_{k+1}^{T}\right]$. 
From the procedure of RU, it can be found that the RU is equivalent to the LMMSE estimator when $N=1$, and when $N \geq 2$, the Jacobian matrix $\boldsymbol{H}_{k+1}^{(i-1)}$ is calculated following the gradient of measurement function continually. Consequently, the overall effectiveness of RU is nonlinear and the nonlinearity is related with measurement function closely.

The iterated Kalman filter presented in [10] behaves in a similar manner but substantially differs from RU. Aiming at finding a $\boldsymbol{x}_{k+1}$ to maximize the quantity $p\left(\mathbf{z}_{k+1} \mid \boldsymbol{x}_{k+1}\right) p\left(\boldsymbol{x}_{k+1}\right)$ which is called maximum a posterior estimation (MAP), the Gauss-Newton measurement update (or iterated measurement update) is derived. In each iteration, current measurement information is all used to update the last iterated state prediction and its contribution is just limited to reducing the error between the sate one-step prediction and the true since the Gauss-Newton is only locally convergent to the MAP estimate for near zero-residual problems. The same question doesn't exist in RU. The innovation of RU is breaking through traditional measurement update ideal of Kalman-typed filters and realizing a nonlinear measurement update on the state. It can be understood in this way that each recursion divides the measurement into $\mathrm{N}$ units and every update is connected with gradient of the measurement function.

Recursive update cubature Kalman filter (RUCKF). In this part, the RU idea is generalized into CT to get RUCKF. Our goal is improving the performance of RU by utilizing the merit of CT which forms a fixed number of deterministically chosen sigma points and then capture the mean and covariance of the state. Here, we define $\boldsymbol{D}_{k+1}=E\left[\left(h\left(\boldsymbol{x}_{k+1}\right)-\hat{\mathbf{z}}_{k+11 k}\right) \boldsymbol{v}_{k+1}^{T}\right]$. Then we can get the optimal filter gain as follows.

$$
\boldsymbol{K}_{k+1}=\left(\boldsymbol{P}_{x z, k+1 \mid k}+\boldsymbol{C}_{k+1}\right)\left(\boldsymbol{P}_{z, k+11 k}+\boldsymbol{D}_{k+1}+\boldsymbol{D}_{k+1}^{T}\right)^{-1} \text {. }
$$

where $\boldsymbol{P}_{z, k+1 \mid k}, \boldsymbol{P}_{x z, k+1 k}$ are the one step prediction covariance of measurement and the one step prediction cross-covariance of state and measurement respectively.

Then, the estimate of state at time $k+1$ is

$$
\hat{\boldsymbol{x}}_{k+1 \mid k+1}=\hat{\boldsymbol{x}}_{k+1 \mid k}+\boldsymbol{K}_{k+1}\left(\mathbf{z}_{k+1}-\hat{\mathbf{z}}_{k+1 \mid k}\right) \text {. }
$$

According to (6), the state posterior estimate error is

$$
\begin{aligned}
\boldsymbol{x}_{k+1}-\hat{\boldsymbol{x}}_{k+1 \mid k+1} & =\boldsymbol{x}_{k+1}-\hat{\boldsymbol{x}}_{k+1 \mid k}-\boldsymbol{K}_{k+1}\left(\boldsymbol{z}_{k+1}-\hat{\boldsymbol{z}}_{k+1 \mid k}\right)=\boldsymbol{x}_{k+1}-\hat{\boldsymbol{x}}_{k+1 \mid k}-\boldsymbol{K}_{k+1}\left(h\left(\boldsymbol{x}_{k+1}\right)+\boldsymbol{v}_{k+1}-\hat{\boldsymbol{z}}_{k+1 \mid k}\right) . \\
& =\boldsymbol{x}_{k+1}-\hat{\boldsymbol{x}}_{k+1 \mid k}-\boldsymbol{K}_{k+1}\left(h\left(\boldsymbol{x}_{k+1}\right)-\hat{\mathbf{z}}_{k+1 \mid k}\right)-\boldsymbol{K}_{k+1} \boldsymbol{v}_{k+1}
\end{aligned}
$$

Based on (7), the state posterior estimate error covariance can be given as follows.

$$
\begin{aligned}
& \boldsymbol{P}_{k+1 \mid k+1}=E\left[\left(\boldsymbol{x}_{k+1}-\hat{\boldsymbol{x}}_{k+1 \mid k+1}\right)\left(\boldsymbol{x}_{k+1}-\hat{\boldsymbol{x}}_{k+1 \mid k+1}\right)^{T} \mid \boldsymbol{Z}_{k+1}\right] \\
& =\boldsymbol{P}_{k+1 \mid k}-\left(\boldsymbol{P}_{x z, k+1 \mid k}+C_{k+1}\right) \boldsymbol{K}_{k+1}^{T}-\boldsymbol{K}_{k+1}\left(\boldsymbol{P}_{x z, k+1 \mid k}+\boldsymbol{C}_{k+1}\right)^{T}+\boldsymbol{K}_{k+1}\left(\boldsymbol{P}_{z, k+1 \mid k}+\boldsymbol{D}_{k+1}+\boldsymbol{D}_{k+1}^{T}\right) \boldsymbol{K}_{k+1}^{T}
\end{aligned}
$$

Note that it is difficult to establish an analytical expression between $\boldsymbol{D}_{k+1}^{(i-1)}$ and $\boldsymbol{D}_{k+1}^{(i)}$ in the recursion. So an approximation is adopted here

$$
\boldsymbol{D}_{k+1}=E\left[\left(h\left(\boldsymbol{x}_{k+1}\right)-\hat{\boldsymbol{z}}_{k+11 k}\right) \boldsymbol{v}_{k+1}^{T}\right]=E\left[\left(h\left(\boldsymbol{x}_{k+1}\right)-\left.h\left(\boldsymbol{x}_{k+1}\right)\right|_{\hat{x}_{k+1 k}}\right) \boldsymbol{v}_{k+1}^{T}\right]=E\left[\boldsymbol{H}_{k+1}\left(\boldsymbol{x}_{k+1}-\hat{\boldsymbol{x}}_{k+1 \mid k}\right) \boldsymbol{v}_{k+1}^{T}\right]=\boldsymbol{H}_{k+1} \boldsymbol{C}_{k+1} \text {. }
$$

where $\boldsymbol{H}_{k+1}=\partial h /\left.\partial \boldsymbol{x}\right|_{x=\hat{x}_{k+1 k}}$.

Combine (5), (8), (9) and the procedure of RU and the system dynamic model formulated in (1)-(2), based on the standard CKF [4], the framework of RUCKF can be summarized as follows.

(1) State prediction

At time $k$, the state $\boldsymbol{x}_{k}$ is assumed to be Gaussian distributed as $\boldsymbol{x}_{k} \sim N\left(\hat{\boldsymbol{x}}_{k \mid k}, \boldsymbol{P}_{k \mid k}\right)$, then the set of $2 n$ sigma points can be chosen and the state is predicted as follows.

$$
\begin{aligned}
& {\left[\chi_{j}\right]_{j=1}^{2 n}=\left[\begin{array}{lll}
\hat{\boldsymbol{x}}_{k \mid k} & \hat{\boldsymbol{x}}_{k \mid k}+\sqrt{(n+\kappa) \boldsymbol{P}_{k \mid k}} & \hat{\boldsymbol{x}}_{k \mid k}-\sqrt{(n+\kappa) \boldsymbol{P}_{k \mid k}}
\end{array}\right] .} \\
& \boldsymbol{\xi}_{j}=f\left(\chi_{j}\right), \quad j=1, \ldots, 2 n . \\
& \hat{\boldsymbol{x}}_{k+1 \mid k}=\frac{1}{2 n} \sum_{j=1}^{2 n} \boldsymbol{\xi}_{j} .
\end{aligned}
$$




$$
\boldsymbol{P}_{k+1 \mid k}=\frac{1}{2 n} \sum_{j=1}^{2 n}\left(\boldsymbol{\xi}_{j}-\hat{\boldsymbol{x}}_{k+1 \mid k}\right)\left(\boldsymbol{\xi}_{j}-\hat{\boldsymbol{x}}_{k+1 \mid k}\right)^{T}+\boldsymbol{Q}_{k} .
$$

where $\chi_{j}$ is the $j$ th column of the matrix $\left[\chi_{j}\right]_{j=1}^{2 n},(\cdot)^{T}$ denotes matrix transpose.

(2) Recursive measurement update

Initialize the recursive parameters before measurement update as follows.

$$
\boldsymbol{C}_{k+1}^{0}=0, \boldsymbol{P}_{k+1 \mid k+1}^{(0)}=\boldsymbol{P}_{k+1 \mid k}, \hat{\boldsymbol{x}}_{k+1 \mid k+1}^{(0)}=\hat{\boldsymbol{x}}_{k+1 \mid k} \text {. }
$$

Then carry out the recursive update steps:

$$
\begin{aligned}
& \text { for } i=1: N \\
& {\left[\xi_{j}^{(i-1)}\right]_{j=1}^{2 n}=\left[\begin{array}{lll}
\hat{\boldsymbol{x}}_{k+1 \mid k+1}^{(i-1)} & \hat{\boldsymbol{x}}_{k+1 \mid k+1}^{(i-1)}+\sqrt{(n+\kappa) \boldsymbol{P}_{k+1 k+1}^{(i-1)}} & \hat{\boldsymbol{x}}_{k+1 k+1}^{(i-1)}-\sqrt{(n+\kappa) \boldsymbol{P}_{k+1 k+1}^{(i-1)}}
\end{array}\right] .} \\
& \gamma_{j}^{(i-1)}=h\left(\boldsymbol{\xi}_{j}^{(i-1)}\right), \quad j=0,1, \ldots, 2 n \text {. } \\
& \hat{\mathbf{z}}_{k+1 \mid k}^{(i-1)}=\frac{1}{2 n} \sum_{j=0}^{2 n} \gamma_{j}^{(i-1)} \text {. } \\
& \boldsymbol{P}_{z, k+1 k k}^{(i-1)}=\frac{1}{2 n} \sum_{j=0}^{2 n}\left(\gamma_{j}^{(i-1)}-\hat{\mathbf{z}}_{k+1 \mid k}^{(i-1)}\right)\left(\gamma_{j}^{(i-1)}-\hat{\mathbf{z}}_{k+1 k}^{(i-1)}\right)^{T}+\boldsymbol{R}_{k+1} . \\
& \boldsymbol{P}_{x z, k+1 \mid k}^{(i-1)}=\frac{1}{2 n} \sum_{j=0}^{2 n}\left(\xi_{j}^{(i-1)}-\hat{\boldsymbol{x}}_{k+11 k+1}^{(i-1)}\right)\left(\gamma_{j}^{(i-1)}-\hat{\mathbf{z}}_{k+11 k}^{(i-1)}\right)^{T} . \\
& \boldsymbol{H}_{k+1 \mid k}^{(i-1)}=\partial h /\left.\partial \boldsymbol{x}\right|_{\boldsymbol{x}=\hat{\boldsymbol{x}}_{k+11 k+1}^{(i-1)}} . \\
& \boldsymbol{K}_{k+1}^{(i)}=\frac{1}{N-i+1}\left(\boldsymbol{P}_{x z, k+1 \mid k}^{(i-1)}+\boldsymbol{C}_{k+1}^{(i-1)}\right)\left(\boldsymbol{P}_{z, k+1 \mid k}^{(i-1)}+\boldsymbol{H}_{k+1}^{(i-1)} \boldsymbol{C}_{k+1}^{(i-1)}+\boldsymbol{C}_{k+1}^{(i-1) T} \boldsymbol{H}_{k+1}^{(i-1) T}\right)^{-1} . \\
& \hat{\boldsymbol{x}}_{k+1 \mid k+1}^{(i)}=\hat{\boldsymbol{x}}_{k+1 \mid k+1}^{(i-1)}+\boldsymbol{K}_{k+1}^{(i)}\left(\boldsymbol{z}_{k+1}-\hat{\mathbf{z}}_{k+1 \mid k}^{(i-1)}\right) \text {. } \\
& \boldsymbol{P}_{k+1 k+1}^{(i)}=\boldsymbol{P}_{k+1 k+1}^{(i-1)}-\left(\boldsymbol{P}_{x z, k+1 k}^{(i-1)}+\boldsymbol{C}_{k+1}^{(i-1)}\right) \boldsymbol{K}_{k+1}^{(i) T}-\boldsymbol{K}_{k+1}^{(i)}\left(\boldsymbol{P}_{x z, k+1 \mid k}^{(i-1)}+\boldsymbol{C}_{k+1}^{(i-1)}\right)^{T}+\boldsymbol{K}_{k+1}^{(i)}\left(\boldsymbol{P}_{z, k+1 \mid k}^{(i-1)}+\boldsymbol{H}_{k+1}^{(i-1)} \boldsymbol{C}_{k+1}^{(i-1)}+\boldsymbol{C}_{k+1}^{(i-1) T} \boldsymbol{H}_{k+1}^{(i-1) T}\right) \boldsymbol{K}_{k+1}^{(i) T} . \\
& \boldsymbol{C}_{k+1}^{(i)}=\left(\boldsymbol{I}-\boldsymbol{K}_{k+1}^{(i)} \boldsymbol{H}_{k+1}^{(i-1)}\right) \boldsymbol{C}_{k+1}^{(i-1)}-\boldsymbol{K}_{k+1}^{(i)} \boldsymbol{R}_{k+1} .
\end{aligned}
$$

where $N$ is the number of recursions which is a user-defined parameter.

Note that the procedure of the measurement update of RUCKF formulated in (14)-(25) is more precise than RUF which is based on EKF. Although the Jacobian matrix $\boldsymbol{H}_{k+1}^{(i-1)}$ is also calculated here, it is only used in the calculation of the cross-covariance of state and measurement noise and the CT can approximate $\boldsymbol{P}_{z, k+1 \mid k}^{(i-1)}, \boldsymbol{P}_{x z, k+1 \mid k}^{(i-1)}$ in a higher precision. Therefore, the RUCKF will outperform the RUF.

\section{Simulation}

Bearing only tracking is a classical model used to test the performance of nonlinear filtering algorithm. In $s-t$ plane, the target moves according to the following second-order linear model.

$$
\boldsymbol{x}_{k+1}=\boldsymbol{F} \boldsymbol{x}_{k}+\boldsymbol{G} \boldsymbol{w}_{k} \text {. }
$$

where $\boldsymbol{x}_{k+1}=[s, \dot{s}, t, \dot{t}]^{T}, s, t$ and $\dot{s}, \dot{t}$ denote target's position and velocity in the $s$ and $t$ directions respectively.

$$
\boldsymbol{F}=\left[\begin{array}{cccc}
1 & T & 0 & 0 \\
0 & 1 & 0 & 0 \\
0 & 0 & 1 & T \\
0 & 0 & 0 & 1
\end{array}\right] \text { and } \boldsymbol{G}=\left[\begin{array}{cc}
\frac{T^{2}}{2} & 0 \\
T & 0 \\
0 & \frac{T^{2}}{2} \\
0 & T
\end{array}\right]
$$

Here, $T$ is the sampling interval. The system noise $\boldsymbol{w}_{k} \square N\left(0, Q \boldsymbol{I}_{2}\right), \boldsymbol{I}_{2}$ is a $2 \times 2$ identity matrix.

The target is observed by radar at the origin of the plane which can provide noisy measurements of the target's bearing 


$$
\mathbf{z}_{k+1}=\arctan \left(t_{k+1} / s_{k+1}\right)+\boldsymbol{v}_{k+1} .
$$

where $\boldsymbol{v}_{k+1} \square N(0, R)$.

Challenge of the target tracking is that the system state is only partially observable according to (27) which makes the posterior probability highly non-Gaussian and traditional Gaussian filters would risk divergence [11]. But here it is intended to choose this application to testify the effect of RU compared with iterate update by taking advantage of its feature.

Simulation parameters are set as follows: $Q=0.012^{2}, R=0.05^{2}, T=1 \mathrm{~s}$, the true initial state is $\boldsymbol{x}_{0 \mid 0}=[-0.05,0.001,0.7,-0.055]^{T}$, the initial estimate is $\hat{\boldsymbol{x}}_{0 \mid 0}=[-0.04,0,0.6,-0.05]^{T}$ with covariance $\boldsymbol{P}_{0 \mid 0}=\operatorname{diag}\left(\left[0.1^{2}, 0.005^{2}, 0.1^{2}, 0.01^{2}\right]^{T}\right)$. For comparison's sake, numbers of RU and iterate update are chosen same $N=20$.

Performance of filters is assessed using the root mean square error (RMSE) of position and velocity defined as follows:

$$
R M S E_{p o s}=\sqrt{\frac{1}{M} \sum_{m=1}^{M}\left(\left(s_{k}^{m}-\hat{\boldsymbol{s}}_{k \mid k}^{m}\right)^{2}+\left(\boldsymbol{t}_{k}^{m}-\hat{\boldsymbol{t}}_{k \mid k}^{m}\right)^{2}\right)} .
$$

where $\left(\boldsymbol{s}_{k}^{m}, \boldsymbol{t}_{k}^{m}\right)$ and $\left(\hat{\boldsymbol{s}}_{k \mid k}^{m}, \hat{\boldsymbol{t}}_{k \mid k}^{m}\right)$ represent the true and estimated position at time $k$ at the $m$ th Monte Carlo run respectively. $M$ is the number of Monte Carlo runs. RMSE of velocity is defined analogously.

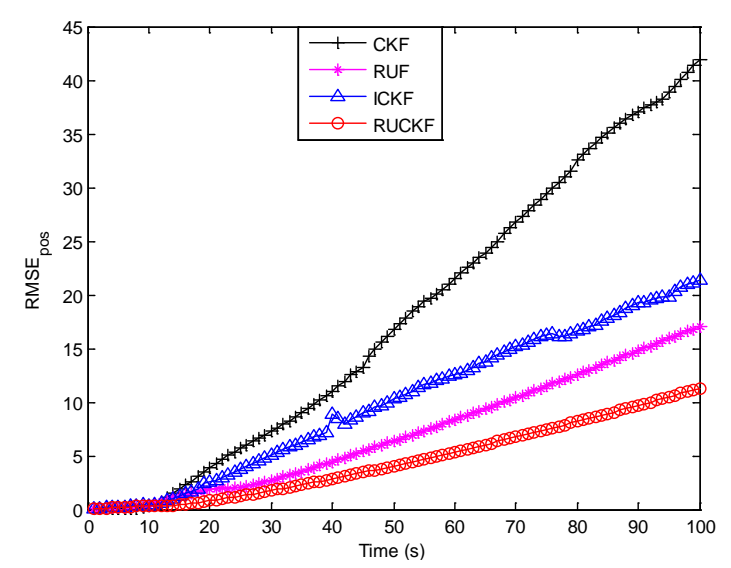

Fig. 1 RMSE of position for CKF, ICKF, RUF and RUCKF

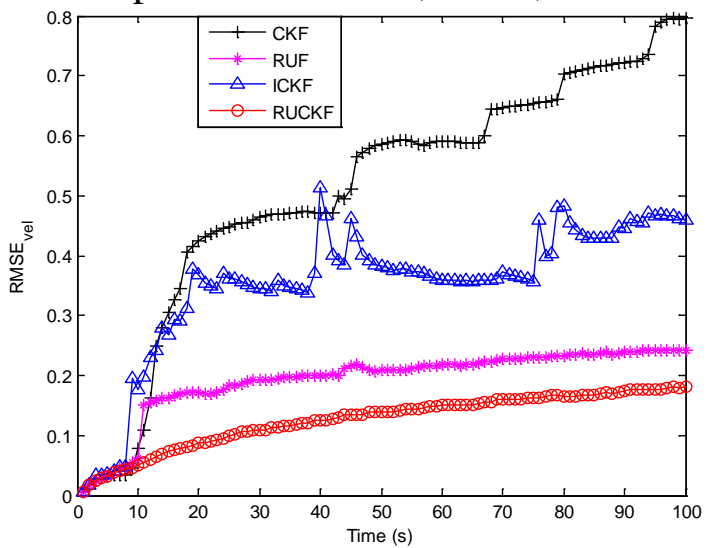

Fig. 2 RMSE of velocity for CKF, ICKF, RUF and RUCKF

100 Monte Carlo experiments have been run and the results are shown in Figs. 1-2. It appears that divergence occurs for CKF which is in response to the partial observability of the system. The highly non-Gaussian posterior probability density introduced by system's measurement function is irreconcilable with the Gaussian assumptions for Gaussian filters and results in terrible performance for CKF. The same situation eases for ICKF. Instead of the Gaussian assumptions, ICKF looks for a MAP estimate for the system and yields a significant improvement over CKF. It is of great surprise that RUF provides amazing performance over CKF and ICKF since RUF is built on EKF and 
subjected the drawbacks of EKF. These facts are sufficed to prove the efficiency of RU idea. Then, there is no doubt about the superior performance for RUCKF shown in Fig. 1-2.

\section{Conclusion}

Recursive update breaks through the traditional measurement update way of Kalman-typed filters and realizes nonlinear update effect on the state. Based on this, a RUCKF is proposed. The proposed algorithm inherits the virtues of RU and fully assimilates the measurement through recursive update procedure. Difference between recursive update and iterate update have been discussed and compared. Simulation results demonstrate the validity and superiority of RUCKF.

\section{Acknowledgement}

This work is supported by the National Natural Science Foundation of China under Grant 61201409 and 61371173; China Postdoctoral Science Foundation under Grant 2013M530147 and 2014T70309; Heilongjiang Postdoctoral Foundation under Grant LBH-Z13052 and LBH-TZ0505; and Fundamental Research Founds for the Central University of Harbin Engineering University under Grant HEUCFQ20150407.

\section{References}

[1] M. R. Morelande, A. F. Garca-Fernndez, Analysis of Kalman filter approximations for nonlinear measurements, IEEE Trans. Signal Process., 61, 22 (2013) 5477-5484.

[2] A. H. Jazwinski, Stochastic Processes and Filtering Theory. New York, NY, USA: Academic 1970.

[3] S. Julier, J. Uhlmann, H. F. Durrant-Whyte, A new method for the nonlinear transformation of means and covariances in filters and estimators, IEEE Trans. Autom. Control, 45, 3 (2000) 477-482.

[4] I. Arasaratnam, S. Haykin, Cubature Kalman filters, IEEE Trans. Autom. Control, 54, 6 (2009) 1254-1269.

[5] I. Arasaratnam, S. Haykin and R. J. Elliott, Discrete-time nonlinear filtering algorithms using Gauss-Hermite quadrature, Proceedings of the IEEE, 95, 5 (2007) 953-977.

[6] M. F. Huber, U. D. Hanebeck, Gaussian filtering for polynomial systems based on moment homotopy, Proceedings of 16th International Conference on Information Fusion, Istanbul, Turkey, July, (2013).

[7] M. B. Luca, S. Azou and G. Burelet al., On exact Kalman filtering of polynomial systems, IEEE Trans. Circuits and System-I: Regular Papers, 53, 6 (2006) 1329-1340.

[8] R. Zanetti, Recursive update filtering for nonlinear estimation, IEEE Trans. Autom. Control, 57, 6 (2012) 1481-1490.

[9] K. Ito, K. Xiong, Gaussian filters for nonlinear filtering problems, IEEE Trans. Autom. Control, 45, 5 (2000) 910-927.

[10] G. Sibley, G. Sukhatme and L. Matthies, The iterated sigma point Kalman filter with applications to long range stereo, Proceedings of Robotics: Science and Systems, Philadelphia, PA, Aug. 2006.

[11]Y. X. Wu, D. W. Hu and M. P. Wu., A numerical-integration perspective on Gaussian filters, IEEE Trans. Signal Process., 54, 8 (2006) 2910-2921. 